\title{
SOME FUNDAMENTAL THEOREMS IN COHOMOLOGY THEORY OF TOPOLOGICAL TRANSFORMATION GROUPS
}

\author{
BY WU-YI HSIANG ${ }^{1}$
}

Communicated by M. H. Protter, January 13, 1971

Ever since the beautiful fixed-point theorem of P. A. Smith for prime periodic transformations on homology spheres, algebraic topologists have been trying to extend his result in the following two directions:

(i) to generalize the transforming groups to general compact Lie groups, and

(ii) to generalize the spaces to those of more complicated cohomologies.

Unfortunately, the past trials are not very successful and the results so obtained are rather disappointing. However, it seems to me that the main reason for such failures is not due to the lack of powerful techniques nor the lack of proper setting, but rather the absence of a suitable viewpoint and a workable approach. As it was pointed out in [5], a perfectly natural approach is to follow the general ideas of I. Schur, E. Cartan and H. Weyl which worked so nicely for the study of linear transformations of compact Lie groups. However, in order to make such a simple minded idea of via "maximal tori" truly workable and penetrating, one needs some exceedingly strong and deep theorems for topological actions of tori so as to fill the missing cornerstone of the Schur lemma for topological transformation groups. The results of [2], [3], [4] clearly demonstrate that one may view the cohomology theory of topological transformation groups as a type of characteristic class theory for fibre bundles with topological $G$-spaces of a given cohomology type as fibre. And the central results that one needs to set up the geometric weight systems will be a certain type of splitting principle for topological actions of tori.

Following A. Borel, we shall denote the twisted product of a $G$ space $X$ and the total space of universal $G$-bundle, $E_{G}$, by $X_{G}$. Namely, $X_{G}=X \times_{G} E_{G}$ is the total space of the universal bundle

$$
X \rightarrow X_{G} \stackrel{\pi}{\rightarrow} B_{G}
$$

1 The author is an Alfred Sloan Fellow and is also supported by N. S. F. grant under the China-U. S. Cooperation Program.

Copyright (c) American Mathematical Society 1971 
with the given $G$-space $X$ as fibre. From the functorial viewpoint of algebraic topology, it is natural and convenient to consider $H^{*}\left(X_{G}\right)$ as the equivariant cohomology algebra of the $G$-space $X$, i.e., $H^{*}\left(X_{G}\right)$ $=H_{G}^{*}(X)$. Then, it is perfectly natural to study the relationships between the various geometric behaviors of the $G$-space $X$ and those algebraic invariants containing in its equivariant cohomology $H_{G}^{*}(X)$. To be precise, let us formulate the following basic problems whose successful solutions will constitute a solid foundation towards the establishment of the "splitting principle."

Problem 1. Let $G$ be a torus group and $X$ be a topological $G$-space with $F$ as its fixed-point set. How does one determine the structure of $H^{*}(F, Q)$ from the structure of $H^{*}\left(X_{G}, Q\right)$ ?

Since the fixed-point set $F(G, X)$ of a general $G$-space $X$ may very well be empty, and the nonexistence of fixed-points tells us almost nothing else, it is essential to consider the whole lattice of connected isotropy subgroups, namely, $\theta^{0}(X)=\left\{G_{x}^{0} ; x \in X\right\}$.

Problem 2. In case $F(G, X)=\varnothing$, how does one determine the set of maximal elements of $\theta^{\circ}(X)$ from the structure of $H^{*}\left(X_{G}, Q\right)$ ?

Problem 3. Let $H_{1}, \cdots, H_{a}$ be the set of maximal elements of $\theta^{\circ}(X)$. How does one compute the cohomology rings of $F\left(H_{j}, X\right)$ as well as $F\left(H_{j}, X\right) / G, j=1, \cdots, a$, from $H^{*}\left(X_{G}, Q\right)$ ?

Problem 4. How does one determine the whole lattice of $\theta^{\circ}(X)$ as well as the cohomology structures of $X_{(I)}$ and $X_{(H)} / G,\left(X_{(H)}=\{x \in X\right.$; $\left.\left.G_{x}^{0}=H\right\}\right)$ for each element $H \in \theta^{\circ}(X)$ ?

We state the answers of the above basic problems as the following theorems of fundamental importance in the cohomology theory of topological transformation groups. Their proofs will appear in a forthcoming paper in Taita Journal of Mathematics. Their far reaching significance and applications will be included in Lecture Notes on Cohomology Theory of Topological Transformation Groups of the author (1970, Fall, National Taiwan University, Taipei, Taiwan).

Notations. In the following, the transforming group $G$ is always a torus group, and $H^{*}\left(B_{G}, Q\right) \simeq Q\left[t_{1}, \cdots, t_{l}\right], l=\operatorname{rk}(G)$, is always abbreviated by $R$. For a given subtorus $K \subseteq G$, we denote $H^{*}\left(B_{K}, Q\right)$ by $R(K)$ and the associated prime ideal $P_{K}=\operatorname{ker}(R \rightarrow R(K))$. For a given $G$-space $X$ and $x \in X$, we shall simply write $R_{x}$ for $R\left(G_{x}^{0}\right)$ and $P_{x}$ for $P_{G_{x}^{0}}$ and $X^{K}=\left\{x \in X ; G_{x}^{0} \supseteq K\right\}, X^{P}=\left\{x \in X ; P_{x} \subseteq P\right\}$ where $P$ is a given prime ideal of $R$. For a given $R$-module $\Re$, we denote the localization of $\mathscr{T}$ at the prime ideal $P$ by $\mathfrak{M}_{P}$. If $P=\{0\}$, then we shall simple denote it by $\widehat{\Re}$.

Theorem 1. Let $\left\{\xi_{1}, \cdots, \xi_{k} ; \nu_{1}, \cdots, \nu_{h}\right\}$ be a generator system of 
the $\hat{R}$-algebra $\hat{H}^{*}\left(X_{G}, Q\right)$ and I be the ideal of defining relations, namely

$$
I \stackrel{\subseteq}{\rightarrow} A \simeq \hat{R}\left[x_{1}, \cdots, x_{k}\right] \otimes \hat{R}_{\hat{R}} \Lambda_{\hat{R}}\left[v_{1}, \cdots, v_{h}\right] \rightarrow \hat{H}^{*}\left(X_{G}, Q\right) \rightarrow 0
$$

is an exact sequence of $\hat{R}$-modules. Then

(i) The radical of $I, \sqrt{ } I$, decomposes into the intersection of s maximal ideals $M_{j}=M\left(\alpha^{(j)}\right)$ whose variety is the point $\alpha^{(j)} \in R^{k}$, i.e.,

$$
\sqrt{ } I=M_{1} \cap \cdots \cap M_{8} .
$$

(ii) The fixed-point set $F$ consists of exactly s connected components, say $F^{j}, j=1, \cdots, s$, and moreover

$$
H^{*}\left(F^{j}, Q\right) \otimes_{Q} \hat{R} \simeq A / I_{j}
$$

where $I_{j}=I_{M_{j}} \cap A$.

(iii) $I=I_{1} \cap \cdots \cap I_{s}=I_{1} \cdot \ldots \cdot I_{s}, I_{j}+I_{1} \cdot \ldots \cdot I_{j-1} \cdot I_{j+1} \cdot \ldots \cdot I_{s}$ $=1$.

Proposition 1. (Criterion for the existence of fixed-point.) The following three conditions are equivalent:

(i) The fixed-point set $F$ is nonempty, i.e., $F \neq \varnothing$.

(ii) There exists a cross-section to the fibration $X_{G \rightarrow B} \rightarrow$.

(iii) The induced map $\pi^{*}: H^{*}\left(B_{G}, Q\right) \rightarrow H^{*}\left(X_{G}, Q\right)$ is injective.

REMARK. The above proposition is false for all noncommutative compact connected Lie groups.

THEOREM 2. Let $J$ be the kernel of $\pi^{*}: R \rightarrow H^{*}\left(X_{G}, Q\right)$ which is clearly a homogeneous ideal of $R$. Let $\sqrt{ } J$ be the radical of $J$ and

$$
\sqrt{ } J=P_{1} \cap \cdots \cap P_{a}
$$

be the irreducible decomposition of $\sqrt{ } J$ into its prime components. Then

(i) Every $P_{j}$ is integral linear in the sense that $P_{j}$ is generated by integral linear elements of $R$. Hence, the variety of $P_{j}, V\left(P_{j}\right)$, is an integral linear subspace of $Q^{l}$ which can be considered as the Lie algebra of a subtorus $H_{j}$ such that $P_{j}$ is exactly the kernel of $R \rightarrow R\left(H_{j}\right)$.

(ii) $\left\{H_{j} ; j=1, \cdots, a\right\}$ are exactly those maximal elements of $\theta^{\circ}(X)$.

(iii) Let $Y^{j}=F\left(H_{j}, X\right), j=1, \cdots, a$. Then

$$
H^{*}\left(Y^{j} / G, Q\right) \otimes_{Q} \hat{R}\left(H_{j}\right) \simeq H^{*}\left(Y_{G}^{j}, Q\right)_{P_{j}} \simeq H^{*}\left(X_{G}, Q\right)_{P_{j}} .
$$

(iv) Let $k=\operatorname{Max}\left(\operatorname{rk}\left(H_{j}\right) ; j=1, \cdots, a\right\}$ and $H_{1}, \cdots, H_{a}$ be those $H_{j}$ with $\mathrm{rk}\left(H_{j}\right)=k$. Then the leading coefficient in the Laurent expansion of the Poincare polynomial of $X_{G}, P\left(X_{G}, u\right)$, at $u=1$, is given by

$$
a_{-k}=2^{-k} \sum_{j=1}^{d} \operatorname{dim}_{Q} H^{*}\left(Y^{j} / G, Q\right) \text {. }
$$


REMARKs. (i) It follows from the equivariant embedding theorem of Mostow, the set of equivariant neighborhoods of $Y=Y^{1}+\cdots+Y^{a}$ possess a countable basis, say $\left\{N_{i}(Y)\right\}$. For technical reasons, we shall consider $H^{*}(X-Y, Q)$ as the limit of $H^{*}\left(X-N_{i}(Y), Q\right)$ and $H^{*}\left(X_{G}-Y_{G}, Q\right)$ as the limit of $H^{*}\left(X_{G}-N_{i}(Y)_{G}, Q\right)$. Then it is clear that

$$
\theta^{0}(X-Y)=\theta^{0}(X) \backslash\left\{H_{j} ; j=1, \cdots, a\right\} .
$$

Hence we may apply Theorem 2 to compute $H^{*}\left(X_{G}-Y_{G}, Q\right)$ and then those maximal elements of $\theta^{0}(X-Y)$. It is clear that this stepwise procedure will give a theoretical solution of Problem 4 in general. However, in many concrete special cases, it is possible to find an easier way out.

(ii) In the above two theorems, we determine the structure of $H^{*}\left(F^{j}, Q\right) \otimes_{Q} \hat{R}$ (resp. $\left.H^{*}\left(F\left(H_{j}, X\right) / G, Q\right) \otimes_{Q} \hat{R}\left(H_{j}\right)\right)$ as an $\hat{R}$-algebra (resp. $\hat{R}\left(H_{j}\right)$-algebra) in terms of generators and relations. In order to actually determine the structure of $H^{*}\left(F^{j}, Q\right)$ (resp. $H^{*}\left(Y^{j} / G, Q\right)$ ), one need only to reduce the given generator system of the $\hat{R}$-algebra $H^{*}\left(F^{j}, Q\right) \otimes_{Q} \hat{R}$ (resp. $\left.H^{*}\left(Y^{j} / G, Q\right) \otimes_{Q} \hat{R}\left(H_{j}\right)\right)$ to a new generator system whose elements lie in $\tilde{H}^{*}\left(F^{j}, Q\right)$ (resp. $\widetilde{H}^{*}\left(Y^{j} / G, Q\right)$ ).

THEOREM 3. It is possible to reduce a generator system $\left\{\eta_{1}, \cdots, \eta_{k}\right\}$ of the $\hat{R}$-algebra $H^{*}\left(F^{j}, Q\right) \otimes_{Q} \hat{R}$ (resp. the $\hat{R}\left(H_{j}\right)$-algebra $H^{*}\left(Y^{j} / G, Q\right)$ $\otimes_{Q} \hat{R}\left(H_{j}\right)$ ) to a new system completely lying in $\tilde{H}^{*}\left(F^{j}, Q\right)$ (resp. $\left.\tilde{H}^{*}\left(Y^{j} / G, Q\right)\right)$ via the following two types of elementary operations:

(i) multiply a generator, say $\eta_{1}$, by an invertible element,

(ii) replace a generator, say $\eta_{1}, b y$

$$
\eta_{1}^{\prime}=\eta_{1}+f\left(\eta_{2}, \cdots, \eta_{k}\right) .
$$

CoRollary 1. If $\hat{H}^{*}\left(X_{G}, Q\right)$ has a generator system of $k$ elements as an $\hat{R}$-algebra, then $H^{*}\left(F^{j}, Q\right)$ has a generator system of at most $k$ elements as a $Q$-algebra for every connected component $F^{j}$ of the fixed-point set $F(G, X)$.

One may further quotient off the part of odd dimensional generators in Theorem 1 to get the following theorem.

TheOREM 4. Let $N=\sqrt{ }\{0\}$ be the ideal of all nilpotent elements of $A$ and $\rho, \rho_{j}$ be the projections of $A$ onto $\hat{H}^{*}\left(X_{G}, Q\right)$ and $H^{*}\left(F_{G}^{\prime}, Q\right)$ relatively. Let $\pi: A \rightarrow A / N \simeq \hat{R}\left[x_{1}, \cdots, x_{k}\right], \tilde{I}=\pi(I), I_{j}=\pi\left(I_{j}\right)$. Then

(i) $\hat{H}^{*}\left(X_{G}, Q\right) / \rho(N) \simeq \hat{R}[X] / \tilde{I}, H^{*}\left(F_{G}^{\prime}, Q\right) / \rho_{j}(N) \simeq \hat{R}[x] / \tilde{I}_{j}$.

(ii) $\sqrt{ } \tilde{I}=\tilde{M}_{1} \cap \cdots \cap \tilde{M}_{s}$ where $\tilde{M}_{j}$ is the maximal ideal of

$$
\alpha^{(j)} \in \hat{R}^{k} \text {. }
$$


(iii) $\sqrt{ } I_{j}=\tilde{M}_{j}, \tilde{I}_{j}=\tilde{I}_{\tilde{M}_{j}} \cap \hat{R}[x]$ and $I=I_{1} \cap \cdots \cap I_{s}, I_{j}+\tilde{I}_{1} \cdot \ldots$ $\cdot \tilde{I}_{j-1} \cdot \tilde{I}_{j+1} \cdot \ldots \cdot \tilde{I}_{s}=1$.

COROLLARY 2. The number of connected components of the fixed-point set $F$ is smaller than or equal to $\operatorname{dim}_{R}\left(\hat{H}^{*}\left(X_{G}, Q\right) / \rho(N)\right)$.

If they happen to be equal, then $H^{*}\left(F^{j}, Q\right)$ are generated by odd dimensional elements for all $j=1, \cdots, s$.

Corollary 3. Suppose that the even part $\left\{\xi_{j}\right\}$ and the odd part $\left\{\nu_{j}\right\}$ are separable, i.e., $\hat{H}^{*}\left(X_{G}, Q\right) \simeq \hat{R}[x] / \tilde{I} \otimes_{R} V$ where $V$ is the subalgebra generated by $\left\{\nu_{j}\right\}$. Then $H^{*}\left(F^{j}, Q\right) \otimes_{Q} \hat{R}$ are also separable and

$$
H^{*}\left(F^{j}, Q\right) \otimes_{Q} \hat{R} \simeq\left\{\hat{R}[x] / \tilde{I}_{j}\right\} \otimes_{\hat{R}} V .
$$

CoRollary 4. If $V \simeq \Lambda_{\hat{R}}\left[v_{1}, \cdots, v_{h}\right]$, then $H^{*}\left(F^{j}, Q\right)$ also contains an exterior algebra of $h$ generators $\Lambda_{Q}\left[\lambda_{1}, \cdots, \lambda_{h}\right]$ such that

$$
\left\{H^{*}\left(F^{j}, Q\right) / I\left(\lambda_{1}, \cdots, \lambda_{h}\right)\right\} \otimes_{Q} \hat{R} \simeq \hat{R}[x] / \tilde{I}_{j} .
$$

Corollary 5. If $\hat{H}^{*}\left(X_{G}, Q\right)$ is generated by odd dimensional elements as an $R$-algebra, then the fixed-point set $F$ is connected and $H^{*}\left(F^{j}, Q\right)$ is also generated by odd dimensional elements.

\section{REFERENCES}

1. A. Borel, et al., Seminar on transformation groups, Ann. of Math. Studies, no. 46, Princeton Univ. Press, Princeton, N. J., 1961.

2. W.-Y. Hsiang, On the geometric weight system of differentiable compact transformation groups on acyclic manifolds, Invent. Math. (to appear).

3. - On the splitting principle and geometric weight system of topological actions. I, Mimes at National Taiwan University (to appear).

4. - On generalization of a theorem of $A$. Borel and their applications in the study of topological actions, Proc. Topology Conf. (Athens, Ga., 1969) (to appear).

5. - On characteristic classes and topological Schur lemma from topological transformation groups viewpoint, Proc. Sympos. Pure Math., vol. 22, Amer. Math. Soc., Providence, R. I., 1971, pp. 105-112.

National Taiwan University, Taipei, Taiwan

University of California, Berkeley, California 94720 